Journal of Patient-Centered

Volume 4

Issue 4 -- Health Disparities and Inequities: Part

Article 26 I

$11-6-2017$

\title{
Six-Year Experience of Influenza Vaccination as a Condition of Employment for a Large Regional Health Care System
}

John R. Brill

Mark Hermanoff

Angela Tonozzi

Mary Jo Capodice

Jennifer Farrar

Zarina Dawoodbhai

Follow this and additional works at: https://aah.org/jpcrr

Part of the Community Health and Preventive Medicine Commons, Health and Medical Administration Commons, Infectious Disease Commons, Influenza Virus Vaccines Commons, Occupational Health and Industrial Hygiene Commons, Public Health Education and Promotion Commons, and the Virus Diseases Commons

\section{Recommended Citation}

Brill JR, Hermanoff M, Tonozzi A, Capodice MJ, Farrar J, Dawoodbhai Z. Six-year experience of influenza vaccination as a condition of employment for a large regional health care system. J Patient Cent Res Rev. 2017;4:262-3.

Published quarterly by Midwest-based health system Advocate Aurora Health and indexed in PubMed Central, the Journal of Patient-Centered Research and Reviews (JPCRR) is an open access, peer-reviewed medical journal focused on disseminating scholarly works devoted to improving patient-centered care practices, health outcomes, and the patient experience. 
Conclusion: Postpartum hypertension is more recognized, and readmissions are becoming more common. We increased efforts to optimize medical management of hypertension and reduce preventable readmissions. Improvement in discharge instructions for patients did not decrease overall admission for postpartum hypertension but may have improved overall patient care. Overall cost analysis would be beneficial to see further economic impact.

\section{Robustness of a Newly Proposed Risk Schema for Lymphatic Dissemination in Endometrioid Endometrial Cancer}

Danielle M. Greer, Jessica J.F. Kram, Callie M. Cox Bauer, Scott A. Kamelle

Aurora University of Wisconsin Medical Group; Center for Urban Population Health; Department of Obstetrics and Gynecology, Brook Army Medical Center; Aurora Gynecologic Oncology

Background: Surgical management for endometrioid endometrial cancer (EEC) includes complete lymph node dissection for all patients at risk of lymphatic dissemination. The standard risk schema, defined by Mayo Clinic, identifies low-risk patients as those with grade $1 / 2$ EEC, myometrial invasion $(\mathrm{MI}) \leq 50 \%$, and tumor diameter (TD) $\leq 2 \mathrm{~cm}$. We recently proposed (and published) a risk schema containing modified forms of grade, MI and TD that suggests a significant decrease in false-negative rate and need for lymphadenectomy in low-risk women.

Purpose: Evaluate robustness of our proposed schema for lymphatic dissemination risk stratification in a subsequent EEC patient cohort. Methods: We retrospectively applied the proposed schema to patients diagnosed with stage I-III EEC during 2014-2015 who underwent pelvic and/or para-aortic lymph node removal. Cancer Registry data were confirmed via chart review. Consistent with the cohort studied during model development, the validation cohort included non-Hispanic white or black patients with complete data describing TD ( $\leq 50 \mathrm{~mm}$ or $>50 \mathrm{~mm})$, MI $(\leq 33 \%,>33 \%$ to $\leq 66 \%$, or $>66 \%$ ) and grade (1 or $2-3)$.

Results: In the validation cohort, 29 (11.7\%) of the 247 EEC patients were node-positive (vs $9.2 \%$ of 737 patients in the development cohort). Risk stratification using the proposed schema produced similar false-positive rates during model development (57.2\%) and validation $(54.6 \%)$, both $20 \%$ lower than when using the standard schema $(76.2 \%$ and $74.3 \%$, respectively). False-negative rates, however, were noticeably different between development and validation cohorts using both the proposed $(0 \%$ and $13.8 \%)$ and standard $(1.47 \%$ and $6.90 \%)$ schemas, suggesting a shift toward lowrisk classification in node-positive patients of the validation cohort. Conclusion: Application of the proposed risk stratification schema to an alternative patient cohort verified the utility of modified risk criteria, including TD with 50-mm cutoff, for identifying low-risk EEC patients who may not require node evaluation. However, in the validation cohort, greater prevalence of lymph node metastasis and low-risk classification of node-positive patients was observed. Discrepancy between cohorts is likely due to greater utilization of sentinel lymph node mapping during the validation period, allowing for increased detection of low-volume metastases. Continued model development and validation is needed, especially to account for the increased sensitivity of new technologies.
FMT Placed by Colonoscopy: Systematic Review and Meta-Analysis

Nadia Huq, Veena Kumaravel, Aboud Affi, Maharaj Singh

Departments of Internal Medicine and Gastroenterology, Aurora Sinai Medical Center; Aurora Research Institute

Background: Fecal transplants are successful in the treatment of recurrent or refractory Clostridium difficile infections (CDI), but there is no consensus on the best method of instillation. Studies have shown greater success with lower gastrointestinal tract placement, but technical aspects of placement are not validated.

Purpose: This review aims to identify common traits and procedural techniques of successful fecal microbiota transplant (FMT) therapy via colonoscopy.

Methods: An electronic search was conducted using OVID Medline and PubMed for articles published from January 2010 to January 2016. The primary outcome of interest was cure by FMT placed via colonoscopy.

Results: Of the 337 articles reviewed, we included 24 studies, from which 11 case reports were excluded from data analysis. The resultant data included 366 patients (64\% female). Point estimate for cure of CDI after FMT for patients over 65 years of age $(9 \%)$ was $84.6 \%$ (95\% confidence interval [CI]: $0.58-0.96 ; \mathrm{P}<0.016)$, cure over the age of 18 with no upper limit specified on age $(74 \%)$ was $85.4 \%$ (95\% CI: $0.76-0.91 ; \mathrm{P}<0.001)$ and, for those identified strictly as $18-65$ years old (17\%), cure was $93 \%$ (95\% CI: $0.83-$ $0.98 ; \mathrm{P}<0.001)$. Patients who stopped antibiotics at least 48 hours prior to FMT (37\%) had a cure rate of $86 \%$ (95\% CI: $0.78-0.91$; $\mathrm{P}<0.001)$ compared to $95 \%(95 \% \mathrm{CI}: 0.90-0.98 ; \mathrm{P}<0.001)$ in patients who stopped antibiotics at least 24 hours prior to FMT $(43 \%)$ and $81 \%(95 \%$ CI: $0.53-0.94 ; \mathrm{P}<0.035)$ in those who stopped less than 24 hours prior to FMT (15\%). In studies that specified use of GoLYTELY ${ }^{\circledR}$ prep prior to colonoscopy (58\%), cure was $91 \%$ (95\% CI: 0.85-0.95; $\mathrm{P}<0.001$ ); whereas those using a split $2-\mathrm{L}$ polyethylene glycol prep (21\%) had $79 \%$ cure $(95 \%$ CI: $0.61-0.90$; $\mathrm{P}<0.004)$. Placement of FMT throughout the colon $(6.8 \%)$ had $96 \%$ cure (95\% CI: $0.77-0.99 ; \mathrm{P}<0.002)$ versus terminal ileum to cecum placement $(59 \%)$ at $88 \%$ cure $(95 \% \mathrm{CI}: 0.78-0.94 ; \mathrm{P}<0.001)$ and cecum to ascending colon $(28 \%)$ at $86 \%$ cure (95\% CI: $0.63-0.95$; $\mathrm{P}<0.006)$. Studies that specified the use of loperamide after FMT $(21 \%)$ had a cure of $85 \%$ (95\% CI: $0.63-0.95$; $\mathrm{P}<0.004)$.

Conclusion: FMT placed by colonoscopy has a role in the cure of recurrent or refractory CDI. Stopping antibiotics 24 hours prior to FMT results in higher percentage cure (95\%). Distribution of FMT throughout the colon has better outcomes than FMT instillation at other locations. Effect of loperamide post-FMT placement is not conclusive due to the low percentage of reported use. Prospective studies are recommended to study these factors for confirmation of effects.

\section{Six-Year Experience of Influenza Vaccination as a Condition of Employment for a Large Regional Health Care System}

John R. Brill, Mark Hermanoff, Angela Tonozzi, Mary Jo Capodice, Jennifer Farrar, Zarina Dawoodbhai

Departments of Population Health, Immunology, Infection Control, Occupational Health, Employee Health, and Practice Optimization, Aurora Health Care 
Background: Influenza remains a significant contributor to morbidity and mortality in the United States. Health care workers (HCW) can be both victims and vectors of influenza. Influenza vaccination of $\mathrm{HCW}$ is protective for both caregivers and patients, but voluntary programs generally fail to achieve rates recommended by the Centers for Disease Control and Prevention. Despite a complex, multifaceted influenza program initiated in 1996 that included significant education and promotion as well as free on-site vaccination, annual Aurora Health Care caregiver immunization rates remained in the mid-70s until adoption of a "condition of employment" strategy in 2011.

Purpose: Discuss the annual effectiveness of the program at achieving caregiver vaccination.

Methods: A steering committee and subgroups meet regularly to evaluate exemption requests, vaccine supply/distribution/ordering, immunization rates and general program overview. In July 2016, an online wellness survey of influenza program perceptions was offered to all Aurora caregivers.

Results: In the 2016-2017 flu season, 97.3\% of HCW were vaccinated. New medical exemption requests have varied from 72 to 127 per year, with a decreasing trend again reflecting the prevalence of permanent exemption. The number of approved annual religious exemptions grew from 39 in 2011 to 64 in 20162017. Since the initial year of implementation, when resignation of $11 \mathrm{HCW}$ was attributed to the vaccination requirement, the number of such resignations has dwindled to 2. Caregiver perceptions: 1,931 caregivers completed the online survey (approximately 6.4\% of all employees). A 5-point Likert scale was used to assess level of agreement with several statements. Caregivers expressed the highest level of agreement with the statement that "the program makes it convenient to get vaccinated" ( $89 \%$ agree or strongly agree) and the lowest level agreement with "the program keeps me healthier" (54\% agree or strongly agree).

Conclusion: An influenza program as a condition of employment leads to high levels of immunization of $\mathrm{HCW}$, with minimal impact on $\mathrm{HCW}$ retention and satisfactory satisfaction among $\mathrm{HCW}$.

\section{Positive Impact on Patient Satisfaction and Caregiver Identification Using Team Facecards: A Quality Improvement Study}

Nicole M. Martin, Khalil Odeh, Lamya Boujelbane, Marvi V. Rijhwani, Susan Olet, Aijaz Noor, Colleen Nichols, Richard Battiola

Department of Internal Medicine, Aurora Sinai Medical Center, Aurora University of Wisconsin Medical Group; Aurora Cardiovascular Services; Aurora Research Institute

Background: Patients satisfaction is an increasingly important metric in measuring the quality of care that hospitals and physicians provide. It can be difficult for patients to remember their providers and their roles, all of which may potentially impact a patient's overall satisfaction.

Purpose: To see if giving facecards with pictures and names of caregivers and description of roles improved patient satisfaction and identification of care team members.

Methods: Team facecards were designed and distributed to the patients during the interventional period of the study. Patients' identification of team members, the role of each physician and overall satisfaction was measured using a longitudinal prospective survey. Patients in the control arm of the study took the same survey but did not receive team facecards.
Results: A total of 192 patients completed the survey. They were divided into a control group $(\mathrm{n}=96,50 \%)$ and an interventional group $(\mathrm{n}=96,50 \%)$ during the period of the study (February 2016-August 2016). Patients who received the intervention were more likely to identify: their team attending (71 [74\%] in the interventional group vs $[34.4 \%]$ in the control group; $\mathrm{P}<0.001)$; team resident (40 $[40.7 \%]$ in the interventional group vs $25[26 \%]$ in the control group; $\mathrm{P}=0.0222)$; team intern (42 [43.8\%] in the interventional group vs $19[19.8 \%]$ in the control group; $\mathrm{P}=0.0004)$. Patients in the interventional group reported slightly higher level of satisfaction (72 $[75 \%]$ reported level of satisfaction $>9$ on a scale of 1 to 10 in the interventional group vs 59 [61.5\%] in the control group).

Conclusion: Use of facecards improves patient identification of primary team members and roles; however, patients still lack enough knowledge of provider roles. Use of facecards showed a slight improvement on overall patient satisfaction.

Relative Associations of Age, Height, and Weight on Sinus of Valsalva and Mid-Ascending Aorta: An Imaging and Epidemiology Study

Mirza Nubair Ahmad, Syed Haris Pir, Mirza Mujadil Ahmad, Sharmeen Hussaini, Bijoy K. Khandheria, A. Jamil Tajik, Khajawa Afzal Ammar

Department of Internal Medicine, Aurora Sinai Medical Center; Aurora Cardiovascular Services

Background: Prior studies show ascending aorta diameter varies with age, height and weight, but they did not evaluate relative influence of these variables on aortic diameter. Since height is genetically determined, and genetic disorders like Marfan syndrome are predominantly associated with sinus of Valsalva (SOV) dilation, we hypothesized height may have stronger association with SOV.

Purpose: Based on anecdotal observation, since age, weight and obesity are acquired attributes, we hypothesized age, weight and body mass index may have greater association with mid-ascending aorta (MAA) diameter, even in normal patients.

Methods: We evaluated echocardiographic studies of patients $\geq 15$ years old that were done in the last 4 years to measure SOV and MAA diameter in normal patients (defined as: medical records and echocardiograms did not reveal any of the 28 aortic dilation risk factors listed in the American Heart Association 2010 guidelines). Results: Of 65,843 patients, 3,201 were identified as normal. SOV measurements were available in 2,046, MAA in 2,334. Age had stronger correlation with MAA $(\beta=0.50 ; \mathrm{r}=0.52 ; \mathrm{P}<0.001)$ than SOV $(\beta=0.33 ; \mathrm{r}=0.35 ; \mathrm{P}<0.001)$. Weight was similarly correlated with diameters of SOV $(r=0.37 ; \mathrm{P}<0.001)$ and MAA $(r=0.37 ; \mathrm{P}<0.001)$. Height had stronger correlation with SOV diameter $(\beta=0.41 ; \mathrm{r}=0.38$; $\mathrm{P}<0.001)$ than MAA $(\beta=0.26 ; \mathrm{r}=0.25 ; \mathrm{P}<0.001)$.

Conclusion: These data suggest that in normal subjects, age, weight and body mass index have stronger associations with the midascending aorta, whereas height has a stronger association with the sinus of Valsalva. 